\title{
Measurement of lung volume and ventilation distribution with an ultrasonic flow meter in healthy infants
}

\author{
A. Schibler*, G.L. Hall", , F. Businger\#, B. Reinmann", J.H. Wildhaber", M. Cernelc", U. Frey"
}

Measurement of lung volume and ventilation distribution with an ultrasonic flow meter in healthy infants. A. Schibler, G.L. Hall, F. Businger, B. Reinmann, J.H. Wildhaber, M. Cernelc, U. Frey. (C) ERS Journals Ltd 2002.

ABSTRACT: Small airway disease in infants is characterised by abnormal lung volume and uneven ventilation distribution. An inert tracer gas washin/washout technique using a pulsed ultrasonic flow meter is presented to measure functional residual capacity (FRC) and ventilation distribution in spontaneously breathing and unsedated infants.

With a pulsed ultrasound sent through the main stream of the flow meter, flow, volume and MM of the breathing gas can be calculated. Sulphur hexafluoride was used as a tracer gas. In a mechanical lung model (volume range 53-188 mL) and in 12 healthy infants (aged $38.3 \pm 9.2$ days; mean $\pm \mathrm{SD}$ ) accuracy and reproducibility of the technique was assessed. Indices of ventilation distribution such as alveolar-based mean dilution number (AMDN) and pulmonary clearance delay (PCD) were calculated.

Mean error of volume measurement in the lung model was $0.58 \%$ (coefficient of variance (CV) $1.3 \%$ ). FRC was in the low predicted range for normal infants $\left(18.0 \pm 2.0 \mathrm{~mL} \cdot \mathrm{kg}^{-1}\right)$ and highly reproducible $(5.5 \pm 1.7 \%$ intra-subject $\mathrm{CV})$. AMDN was $1.63 \pm 0.15$ and PCD was $52.9 \pm 11.1 \%$.

Measurement of functional residual capacity and ventilation distribution using a sulphur hexafluoride washin/washout and an ultrasonic flow meter proved to be highly accurate and reproducible in a lung model and in healthy, spontaneously breathing and unsedated infants.

Eur Respir J 2002; 20: 912-918.
Swiss Paediatric Respiratory Physiology Research Group: *Paediatric Intensive Care Unit, " Division of Paediatric Respiratory Medicine and Dept of Paediatrics, University of Bern, and Dept of Respiratory Medicine, University Children's Hospital, Zürich, Switzerland.

Correspondence: A. Schibler, Paediatric Intensive Care Unit, Dept of Paediatrics, Children's Hospital University of Bern, 3010 Bern, Switzerland. Fax: 41316329468

E-mail: andreas.schibler@insel.ch

Keywords: Functional residual capacity, infant lung function, lung model, ultrasonic flow meter, ventilation distribution

Received: March 152001

Accepted after revision: March 222002

This study was supported by the Swiss National Foundation (SCORE 32-51974.97), Australian National Health and Medical Research Council, Swiss CF Foundation, AstraZeneca (Switzerland)
Infants with small airways disease such as wheezing disorders, cystic fibrosis and chronic lung disease have hyperinflated lungs and ventilation inhomogeneities $[1,2]$. In infants it is difficult to target small airways function. The rapid thoracic compression technique and whole-body plethysmography have been proposed to provide a measure of small airways function, however these methods have limited capacity, require sedation and might be not suitable for larger epidemiological studies.

To quantify the degree of functional abnormalities of the lung and to study the therapeutic effect of inhaled bronchodilators or topical anti-inflammatory drugs, an accurate and easy-to-use technique to measure lung volume and ventilation distribution in infants is necessary. This can be achieved by multiple breath gas washout techniques. Gas dilution techniques determine functional residual capacity (FRC) by measuring areas of the lung that readily communicate with the central airways during tidal breathing. Two gas dilution techniques frequently used are closed helium $(\mathrm{He})$ dilution and open-circuit multiple-breath nitrogen washout (MBNW). Although MBNW can be performed easily, the considerable change in gas viscosity during the washout manoeuvre significantly affects the accuracy of the gas flow $\left(V^{\prime}\right)$ measurement by pneumotachography $[3,4]$. The He dilution technique avoids this latter problem, but is based on a closed circuit and leaks in the measuring system reduce its accuracy [5]. In addition the He technique has a long time constant and cannot be used to assess gas mixing. Sulphur-hexafluoride $\left(\mathrm{SF}_{6}\right)$ washout is a potentially suitable technique for children, including those who require near $100 \%$ oxygen $\left(\mathrm{O}_{2}\right)$ to maintain normal haemoglobin saturation $[6,7]$.

The aim of this study was to develop a simple technique able to measure lung volume (FRC) and ventilation inhomogeneities in spontaneously breathing infants without altering the infant's breathing and reducing problems due to leak. The aim was to validate the accuracy and feasibility of a new $\mathrm{SF}_{6}$ washin/washout technique in a mechanical lung model and in unsedated healthy infants. 


\section{Material and methods}

\section{Subjects}

Twelve healthy infants (four female, eight male), aged $38.3 \pm 9.2$ days (mean \pm SD) were measured. The infants had no reported respiratory events prior to study enrolment, were born at term, and had no parental history of asthma or smoking. The study was approved by the ethics committee of the University Children's Hospital, Berne and written parental informed consent was obtained before measurements.

\section{Study design}

The $\mathrm{SF}_{6}$ washin/washout technique was initially evaluated in a mechanical lung model and subsequently tested in nonsedated spontaneously breathing, healthy infants. Measured FRC and indices of ventilation distribution were compared to reported results in the literature.

\section{Sulphur hexafluoride multiple-breath washin/washout system}

FRC and ventilation indices were measured with a multiple-breath washin/washout technique using $\mathrm{SF}_{6}$ as a tracer gas. The system used was adapted from a previous study of FRC in ventilated children and animals [8]. The system (fig. 1) included a computer, a constant bypass flow $\left(8-12 \mathrm{~L} \cdot \mathrm{min}^{-1}\right)$, an ultrasonic flow meter (Spiroson (B) Scientific; ECO Medics AG, Dürnten, Switzerland) and an airtight face mask. A pressure driven blender, controlled by the computer was used to switch from room air to a gas mixture containing room air and $4 \% \mathrm{SF}_{6}$. Inspiratory and expiratory $V^{\prime}$ were measured with the ultrasonic flow meter and integrated to calculate inspired and expired volumes $(V)$. Flow is determined by measuring the transit time of a pulsed ultrasound travelling through the streaming medium. The sound is either accelerated or de-accelerated by the movement of the medium, causing, for a fixed distance across the medium, a

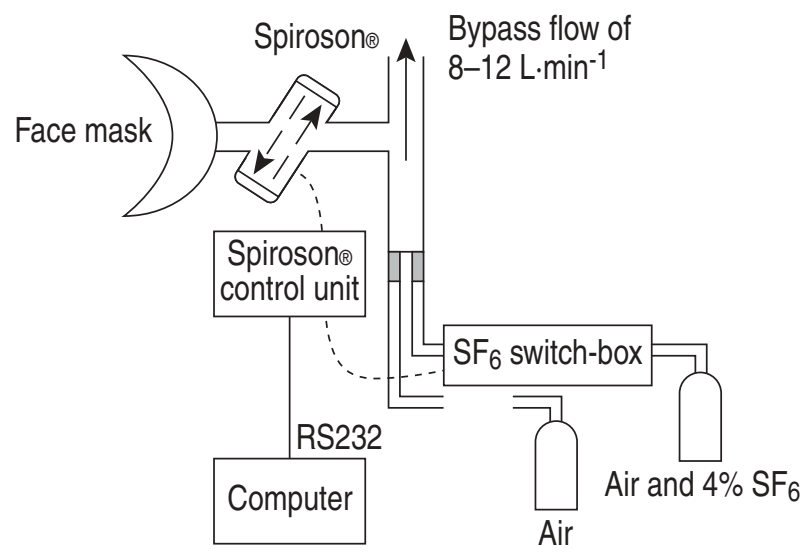

Fig. 1.-Configuration of the sulphur hexafluoride $\left(\mathrm{SF}_{6}\right)$ washin/ washout equipment. decrease in the downstream and an increase in the upstream transit time. This change in transit time is related to gas velocity. The molar mass ( $\mathrm{MM}$ in $\mathrm{g} \cdot \mathrm{mol}^{-1}$ ) of the gas in the main stream of the flow meter can be computed from the transit time with high accuracy and its value is directly proportional to the density of the medium. The MM can be assessed with the Spiroson $\mathbb{R}$ ultrasonic flow meter over the range of molar mass of $20-45 \mathrm{~g} \cdot \mathrm{mol}^{-1}$ with a precision of $0.01 \mathrm{~g} \cdot \mathrm{mol}^{-1}$. Hence using the formula:

$$
\varphi=(\mathrm{MM} \cdot \mathrm{p}) /(\mathrm{R} \cdot \mathrm{T})
$$

the MM can be calculated (where $\varphi$ is the gas density, $\mathrm{MM}$ is the molar mass, $\mathrm{p}$ is the pressure, $\mathrm{R}$ is the gas constant and $\mathrm{T}$ is the temperature). However the precise temperature along the sound transmission path must be determined. Due to the complexity of the determination of the temperature along the sound transmission path and because minor changes in temperature are present during tidal breathing, the changes of temperature along the sound path can be simulated using a temperature model. The temperature model uses the measured flow, instrumental dead space and the preset temperature (at body temperature and ambient pressure, and saturated with water vapour (BTPS) correction parameters) of the inspiratory and expiratory gas to calculate the temperature changes along the sound transmission path. The composition of the inspiratory gas differs from the expiratory due to pulmonary gas exchange. Normally the inspiratory gas constitutes $21 \% \mathrm{O}_{2}$ and $79 \%$ nitrogen $\left(\mathrm{N}_{2}\right)$ resulting in a MM of $28.85 \mathrm{~g} \cdot \mathrm{mol}^{-1}$. During expiration the MM is slightly higher due to the presence of carbon dioxide $\left(\mathrm{CO}_{2}\right)$, the $\mathrm{MM}$ of which is $\sim 29.09 \mathrm{~g} \cdot \mathrm{mol}^{-1}$. To take account of these effects of temperature and gas composition on the MM signal, the authors subtracted from the measured MM during washin and washout, the baseline MM which was obtained during the corresponding phase of tidal breathing prior to the $\mathrm{SF}_{6}$ washin (assuming that during a measurement the breathing conditions remain constant). The difference between measured and baseline $\mathrm{MM}$ is called $\triangle \mathrm{MM}$. The washin or washout curve of $\mathrm{SF}_{6}$ was described using $\triangle \mathrm{MM}$. To obtain the instantaneous $\mathrm{SF}_{6}$ fraction at every moment during washin and washout, $\triangle \mathrm{MM}$ was divided by the $\mathrm{MM}$ of $\mathrm{SF}_{6}\left(146.05 \mathrm{~g} \cdot \mathrm{mol}^{-1}\right)$. A twopoint calibration of the MM signal was performed using room air and $4 \% \mathrm{SF}_{6}$.

An infant ultrasonic flow meter (dead space of $1.3 \mathrm{~mL}$ ) was used for measurements in infants. A medium ultrasonic flow meter (dead space of $3.2 \mathrm{~mL}$ ) with a larger inner diameter was used in the lung model due to the higher flow encountered when the model was ventilated at a rate of $60 \mathrm{~min}^{-1}$ and with a tidal volume $(V \mathrm{~T})$ of $80 \mathrm{~mL}$. The flow meter was calibrated with a high precision calibration syringe (Hans Rudolph, 5510 Series; Gambro AG, Hünebach, Switzerland) and considered to be calibrated adequately if integrated volume was within $1 \%$ of $100 \mathrm{~mL}$. The authors ensured the flow head was airtight up to a pressure of $20 \mathrm{cmH}_{2} \mathrm{O}$. The $\mathrm{MM}$ of the in- and exhalation gas was measured with the 


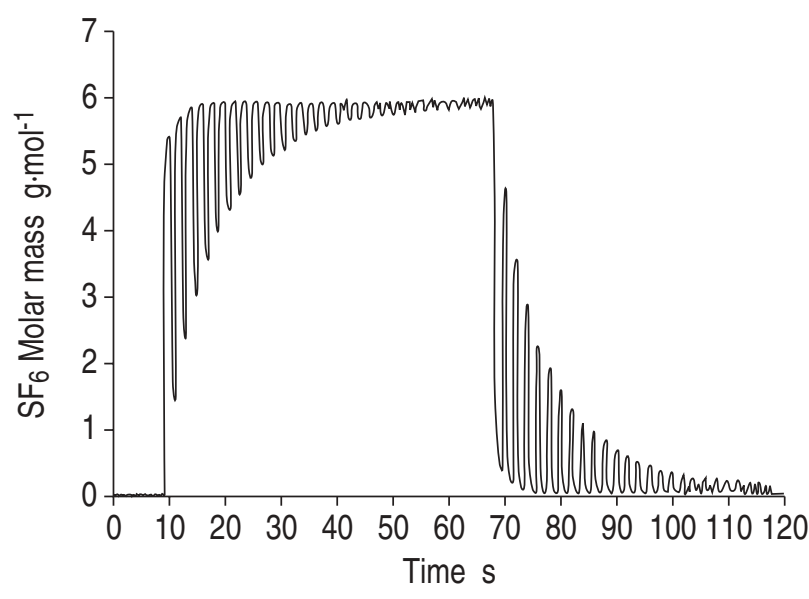

Fig. 2.-A typical washin and washout of sulphur hexafluoride $\left(\mathrm{SF}_{6}\right)$. Washout of $\mathrm{SF}_{6}$ is initiated when end expiratory $\mathrm{SF}_{6}$ concentration is constant.

ultrasonic flow meter (fig. 2). The signals of $V^{\prime}$ and MM were acquired by the Spiroson control unit, digitised and sampled at a rate of $200 \mathrm{~Hz}$. The digitised signals were processed and stored on the computer.

\section{Calculation of functional residual capacity and indices of ventilation distribution}

The FRC of washout was obtained by dividing the measured expired $\mathrm{SF}_{6}$ volume with the end-tidal $\mathrm{SF}_{6}$ concentration before washout. The initial $\mathrm{SF}_{6}$ volume within the flow meter and the connectors was subtracted from the total of the exhaled $\mathrm{SF}_{6}$ volume. The FRC of the washin was determined similarly using the mathematical mirror image of the washout signal. FRC was measured in duplicate for each washin/washout. In each infant at least three $\mathrm{SF}_{6}$ washin/washouts were performed and the six FRC values were calculated. $V^{\prime}, V$ and FRC were corrected to BTPS conditions. The calculation of the washout indices was limited to the most frequently reported and calculated as described by the original authors. The lung clearance index (LCI) is the number of volume turnovers required to reduce end-tidal $\mathrm{SF}_{6}$ concentration to one-fortieth of the tracer concentration at the start of the washin/washout [9]. The alveolar-based mean dilution number (AMDN) is the ratio between the first and the zero moments of the washout curve. The number of volume turnovers was calculated using the cumulative expired alveolar volume ( $V \mathrm{~T}$ minus dead space volume) [10]. The mean dilution number (MDN) was calculated similarly to AMDN but the cumulative expired alveolar volume is not corrected for Fowler dead space [3]. The pulmonary clearance delay (PCD) is defined as $100 \times$ (actual average time a tracer gas remains in the lungs the ideal time)/the ideal time [9]. The mixing ratio is the ratio between the observed and the ideal number of breaths required reducing the end-tidal tracer gas concentration to one-fortieth of the concentration at the start of the washout [11]. A low value of any index indicates even ventilation.
The Fowler dead space $(V D)$ was obtained from the first three expirations during washout [9]. $V \mathrm{D}$ was defined as the volume expired when the $\mathrm{SF}_{6}$ concentration reached $50 \%$ of the end-tidal $\mathrm{SF}_{6}$ concentration of that breath. At least four $V \mathrm{~T}$ prior to and following the $\mathrm{SF}_{6}$ washin were recorded to evaluate if the breathing pattern changes during the $\mathrm{SF}_{6}$ washin.

FRC and ventilation indices were calculated using the software provided with the ultrasonic flow meter and the authors' own algorithms written in LabView 5.0.

\section{Measurements in infants}

$\mathrm{SF}_{6}$ washin/washout was performed during regular sleep without sedation and in supine position. The transcutaneous haemoglobin saturation was continuously monitored during the measurement (Ohmeda 3800 Pulse Oximeter, Helsinki, Finland). A face mask, sealed around the nose and mouth to ensure an airtight fit, was carefully manipulated into place for the measurements. The face mask had an effective dead space of $5 \mathrm{~mL}$ (measured by water displacement). At least three washin/washout of $\mathrm{SF}_{6}$ were performed in every infant.

\section{Lung model}

The lung model has been described previously [12]. Shortly summarised, a linear pump (Scireq Inc., Montreal, Canada) with a precision of $0.5 \%$ (full stroke), enabling the simulation of tidal and forced flow patterns was used [13]. The $V \mathrm{~T}$ can be reproduced with a precision of $\pm 1 \%$ at frequencies between 10 120 beats per minute (bpm). The linear pump was connected to a series of known volumes $(55,60,80$, $90,95,110,115,120,125,130,135,140$ and $185 \mathrm{~mL})$. In the present experiment the lung model was washed out using breathing frequencies of 20,40 and $60 \mathrm{bpm}$ and $V \mathrm{~T}$ of 24, 34, 40, 49, 56, 68, 74 and $79 \mathrm{~mL}$. The ratio of $V \mathrm{~T}$ to FRC was chosen to be between $0.4-0.6$. The volumes were built of copper and filled with copper wool in order to maximise and homogenise the heat dissipation. The exact volumes of these elements were calculated from water displacement as well as from their physical dimensions, in which both copper wool and connector volumes were taken into account. A minimal leak of the lung model was observed using a pressure up to $20 \mathrm{cmH}_{2} \mathrm{O}$. This leak was dependent on pressure as well as frequency and $V \mathrm{~T}$. To quantify the effect of the leak on the accuracy of FRC measurement the following set-up was used: the lung model was flushed with a mixture of room air $4 \% \mathrm{SF}_{6}$. An empty rubber balloon was attached to the distal end of the ultrasonic flow meter. The linear pump then ventilated the lung model at different frequencies (20 to $60 \mathrm{bpm})$ and the $V \mathrm{~T}$ were adjusted to have a $V \mathrm{~T} / \mathrm{FRC}$ ratio of 0.4 and 0.6 . The empty highly compliant rubber balloon was needed to achieve a closed system without adding dead 
space and to keep the pressure within the system similar to those encountered during FRC measurements in infants. Therefore the balloon served as a reservoir to ensure an approximately constant volume low pressure closed system over the whole breath cycle. The MM signal was then measured over $260 \mathrm{~s}$ (approximately five times longer than the average washin/ washout time of the lung model). Any decrease of the $\mathrm{MM}$ indicated a loss of $\mathrm{SF}_{6}$ and hence allowed the magnitude of a leak to be quantified.

\section{Statistics}

For each parameter and set of six measurements (three washin and three washout) the mean \pm SD were calculated. Reproducibility of the system in vitro and in vivo was evaluated by calculating the coefficient of variation (CV). The accuracy of the measurements (limits of agreement) in the mechanical lung model was assessed with the difference between the measured volume and the mean of measured and preset volumes [14]. To examine the effect of $V \mathrm{~T}$ on the accuracy of FRC measurements in the lung model a two-way analysis of variance was used. Per cent error was defined as: $100 \times$ absolute value of (preset volumemeasured volume)/preset volume. Differences between volume measurements in the lung model were assessed using a paired t-test. A p-value $<0.05$ was considered to be significant.

\section{Results}

\section{Lung model}

Bench tests in the mechanical lung model showed highly accurate volume measurements using the $\mathrm{SF}_{6}$ gas washin/washout technique. The FRC measurement error was $0.62 \pm 1.49 \mathrm{~mL}$ (range: $0-3.66 \mathrm{~mL}$ ), with a $\mathrm{CV}$ of $1.3 \%$ and the error expressed in percent was $0.58 \pm 1.3 \%$ (range: $0-3.2 \%$ ). The Altmann and BLAND [14] plot in fig. 3 shows the agreement between the measured and known volume of the mechanical lung model. Changes in $V \mathrm{~T}$ or respiratory rate did not affect the accuracy of volume measurement in the lung model $(\mathrm{p}=0.46)$. Table 1 compares the results with similar bench tests for measurements reported in the literature. The maximal leak was observed at a breathing frequency of $60 \mathrm{bpm}$ and a $V \mathrm{~T}$ of $74 \mathrm{~mL}$. The MM signal decreased at these settings at a rate of $0.014 \mathrm{~g} \cdot \mathrm{mol}$ per $\mathrm{min}^{-1}(0.24 \%$ of the observed endwashin $\mathrm{SF}_{6}$ concentration) or with $0.00052 \mathrm{~g} \cdot \mathrm{mol}$ per breath $^{-1}(0.009 \%)$. The authors therefore compensated the measured FRC data for the measured leak of the lung model. The precision of the measurement was within recommended measurement variability specified by the recent ERS-standards for infant lung function equipment $[17,18](<2.5 \%$ for infant lung volumes between 50 and $300 \mathrm{~mL}$, minimal $2 \mathrm{~mL}$ ).

\section{Healthy infants}

Biometric data, FRC, and indices of ventilation distribution are shown in Table 2. For each infant a

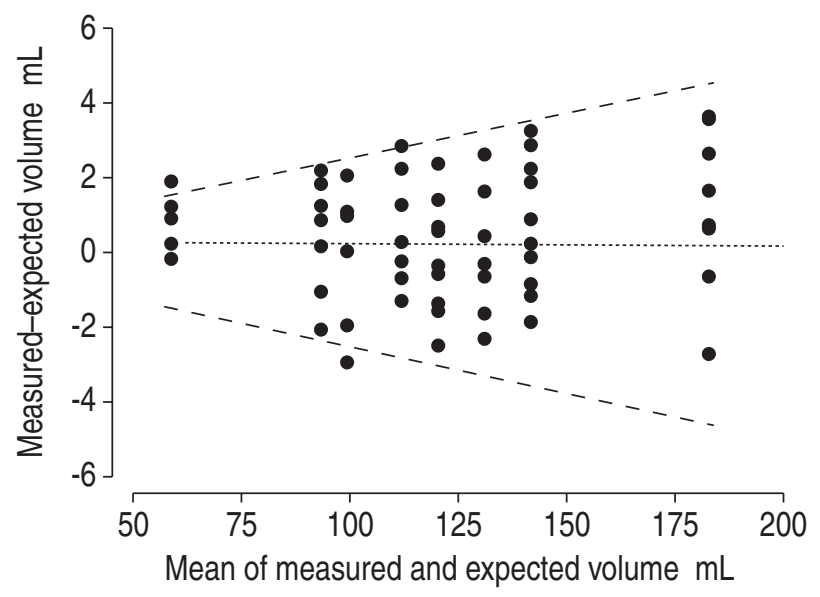

Fig. 3.-Accuracy of in vitro assessment of lung volume. The average of the measured and known volume of a mechanical lung model is plotted against the differences. The dashed lines indicate the 95\% confidence interval between the expected and the measured volumes (European Respiratory Society standards). …....... mean.

Table 1.-Accuracy of volume measurements in mechanical lung models reported in the literature

\begin{tabular}{lccll}
\hline $\begin{array}{l}\text { First author } \\
\text { [ref. no.] }\end{array}$ & $\begin{array}{c}\text { Volume range } \\
\mathrm{mL}\end{array}$ & Error & Range & $\mathrm{CV}$ \\
\hline SCHIBLER [15] & $15-670$ & 1.95 & $0-10.7$ & 2.5 \\
SCHULZE [6] & $7-70$ & 0.70 & $0-7.8$ & \\
SIVAN [16] & $100-500$ & 2.30 & $0-11$ & 2.3 \\
VILSTRUP [7] & $5-60$ & 2.40 & $2-6$ & 4 \\
SCHIBLER [8] & $10-60$ & 0.59 & $0-9.3$ & 4.3 \\
Present study & $53-188$ & 0.58 & $0-3.2$ & 1.3 \\
\hline
\end{tabular}

Data are presented as $\%$ unless otherwise stated. $\mathrm{CV}$ : coefficient of variance.

minimum of six FRC (three washin and three washout) calculations were made. The mean $\mathrm{CV}$ for FRC during spontaneous breathing was $5.5 \pm 1.6 \%$ (range: 1.7-7.3\%). FRC measured during washin showed no significant difference from FRC measured during washout $(\mathrm{p}=0.52)$, nor was there any change in tidal breathing during the washin or washout of $\mathrm{SF}_{6}$, $V \mathrm{~T}$ prior to washin was $28.9 \pm 6.6 \mathrm{~mL}$ and $V \mathrm{~T}$ at the end of washin was $29.7 \pm 6.8 \mathrm{~mL}$. The $V \mathrm{~T} / \mathrm{FRC}$ ratio was within normal range $(0.4-0.6)$. Table 3 compares the ventilation indices with reported values in the literature.

\section{Discussion}

The measurement of lung volume (FRC) and ventilation inhomogeneities in infants with small airway disease is important to monitor the effects of therapeutic interventions such as bronchodilators and inhaled topical steroids. The breath-by-breath inert gas washout method provides a unique opportunity to diagnose such conditions without radiation or open lung biopsy or at least reduce the use of such methods. A new $\mathrm{SF}_{6}$ washin/washout technique to measure 
Table 2. - Functional residual capacity (FRC) and measured ventilation distribution indices in 12 healthy infants

\begin{tabular}{|c|c|c|c|c|c|c|c|c|c|c|c|c|c|c|}
\hline $\begin{array}{l}\text { Sub- } \\
\text { ject } \\
\text { no. }\end{array}$ & $\begin{array}{l}\text { Weight } \\
\text { kg }\end{array}$ & $\begin{array}{c}\mathrm{FRC} \\
\mathrm{mL} \cdot \mathrm{kg}^{-1}\end{array}$ & $\begin{array}{c}\mathrm{CV} \\
\%\end{array}$ & $\begin{array}{c}\text { FRC } \\
\text { pred } \\
\mathrm{mL} \cdot \mathrm{kg}^{-1}\end{array}$ & LCI & $\begin{array}{l}\mathrm{CV} \\
\%\end{array}$ & AMDN & $\begin{array}{c}\mathrm{CV} \\
\%\end{array}$ & MDN & $\begin{array}{c}\mathrm{CV} \\
\%\end{array}$ & $\underset{\%}{P C D}$ & $\begin{array}{l}\text { CV } \\
\%\end{array}$ & MR & $\begin{array}{c}\text { CV } \\
\%\end{array}$ \\
\hline 1 & 5.3 & 16.9 & 6.7 & 19.6 & 6.9 & 13.6 & 1.9 & 6.3 & 2.8 & 10.1 & 56.2 & 28.0 & 1.6 & 10.1 \\
\hline 2 & 4.1 & 19.6 & 5.0 & 26.4 & 4.5 & 31.6 & 1.4 & 9.5 & 2.1 & 12.7 & 51.7 & 25.6 & 1.5 & 8.7 \\
\hline 3 & 4.3 & 18.6 & 3.2 & 19.4 & 5.4 & 39.2 & 1.4 & 25.7 & 2.2 & 14.8 & 53.4 & 19.2 & 1.5 & 6.7 \\
\hline 4 & 5.3 & 15.5 & 7.3 & 19.6 & 6.3 & 11.2 & 1.6 & 3.0 & 2.4 & 1.0 & 52.6 & 3.8 & 1.5 & 1.3 \\
\hline 5 & 5.1 & 16.4 & 4.8 & 20.6 & 5.6 & 17.5 & 1.5 & 10.0 & 2.2 & 8.3 & 54.1 & 31.7 & 1.5 & 11.2 \\
\hline 6 & 3.9 & 21.2 & 4.0 & 24.7 & 5.1 & 14.0 & 1.3 & 4.8 & 2.2 & 5.3 & 58.2 & 25.4 & 1.6 & 9.3 \\
\hline 7 & 3.6 & 18.5 & 3.1 & 25.6 & 6.7 & 5.9 & 1.7 & 4.1 & 2.4 & 4.4 & 53.6 & 6.3 & 1.5 & 2.2 \\
\hline 8 & 4.3 & 21.1 & 3.8 & 22.5 & 7.1 & 12.7 & 1.7 & 7.4 & 2.5 & 7.0 & 41.8 & 18.8 & 1.4 & 5.5 \\
\hline 9 & 4.8 & 14.2 & 6.9 & 21.2 & 6.1 & 21.4 & 1.6 & 13.0 & 2.3 & 14.1 & 53.0 & 7.9 & 1.5 & 2.7 \\
\hline 10 & 4.3 & 18.2 & 7.3 & 22.5 & 5.7 & 23.3 & 1.5 & 16.9 & 2.5 & 17.2 & 54.2 & 10.8 & 1.5 & 3.8 \\
\hline 11 & 3.6 & 16.1 & 6.9 & 23.1 & 5.2 & 37.5 & 1.4 & 18.0 & 2.1 & 8.0 & 65.5 & 27.3 & 1.7 & 10.8 \\
\hline 12 & 4.8 & 15.5 & 6.7 & 20.5 & 6.7 & 7.7 & 1.6 & 6.1 & 2.5 & 5.0 & 47.9 & 14.0 & 1.5 & 4.5 \\
\hline Mean & 4.4 & 18.0 & 5.5 & 22.1 & 6.0 & 19.6 & 1.6 & 10.4 & 2.4 & 9.8 & 52.9 & 18.2 & 1.5 & 6.4 \\
\hline SD & 0.6 & 2.0 & 1.7 & $2.4^{\#}$ & 1.3 & 11.2 & 0.2 & 6.8 & 0.3 & 6.5 & 11.1 & 9.5 & 0.1 & 3.5 \\
\hline $\mathrm{CV}$ & & 11.4 & & 11.0 & 21.7 & & 13.9 & & 13.5 & & 21.1 & & 7.3 & \\
\hline
\end{tabular}

$\mathrm{CV}$ : coefficient of variation; FRC mL $\mathrm{kg}^{-1}$ : FRC corrected for body weight measured with sulphur hexafluoride $\left(\mathrm{SF}_{6}\right)$ washin/washout; FRC pred $\mathrm{mL} \cdot \mathrm{kg}^{-1}$ : predicted FRC values from collated data in Ref [19]; LCI: lung clearance index; AMDN: alveolar-based mean dilution number; MDN: mean dilution number; PCD: pulmonary clearance delay; MR: mixing ratio. ${ }^{\#}$ : $\mathrm{p}=0.0001$ versus $\mathrm{FRC} \mathrm{mL} \cdot \mathrm{kg}^{-1}$.

Table 3.-Comparison of ventilation distribution indices reported in the literature

\begin{tabular}{|c|c|c|c|c|c|c|}
\hline Subjects & First author [ref. no.] & Subjects $n$ & LCI & AMDN & MDN & PCD \% \\
\hline Infants with lung disease & SCHIBLER [15] & 23 & $5.6 \pm 1.9$ & $2.2 \pm 0.4^{\#}$ & $2.3 \pm 0.6$ & \\
\hline $\begin{array}{l}\text { Ventilated children with lung } \\
\text { disease }\end{array}$ & SCHIBLER [20] & 22 & $9.5 \pm 1.7 * * *$ & $2.0 \pm 0.3^{* * *}$ & $2.5 \pm 0.4$ & $75 \pm 24^{*}$ \\
\hline Healthy children & KRAEMER and MEISTER [21] & 17 & $7.7 \pm 1.1 * * *$ & & $2.2 \pm 0.3^{* *}$ & \\
\hline Healthy preterm infants & SHAO [22] & 20 & $10.8 \pm 1.4 * * *$ & & $2.2 \pm 0.2^{\#}$ & \\
\hline Healthy infants & Present study & 12 & $6.2 \pm 0.9$ & $1.6 \pm 0.2$ & $2.4 \pm 0.3$ & $52.9 \pm 11.1$ \\
\hline
\end{tabular}

Data are presented as mean \pm SD unless otherwise indicated. LCI: lung clearance index; AMDN: alveolar-based mean dilution number; MDN: mean dilution number; PCD: pulmonary clearance delay. Significantly different compared to the present study: *: $\mathrm{p}<0.05 ; * *: \mathrm{p}<0.01 ;{ }^{\#}: \mathrm{p}<0.005 ; * * *: \mathrm{p}<0.001$.

FRC and ventilation distribution was validated in a mechanical lung model and in unsedated healthy, spontaneously breathing infants. An ultrasonic flow sensor was used to simultaneously measure $V^{\prime}, V$ and the tracer gas concentration. High accuracy and reproducibility of the measurement technique could be demonstrated in a mechanical lung model. SF6 washin/washout measurements could be easily obtained in all infants, and reproducible FRC and ventilation distribution data calculated.

Any gas dilution technique to measure FRC has intrinsic limitations. He dilution can determine FRC highly accurately, provided there is no leak in the system. The technique is limited, however, as $\mathrm{He}$ analysers are sensitive to gases, other than $\mathrm{He}$, such as $\mathrm{CO}_{2}, \mathrm{O}_{2}, \mathrm{~N}_{2}$, and water vapour. In addition, it is not possible to assess ventilation distribution of the lung with the $\mathrm{He}$ dilution technique as the end-tidal $\mathrm{He}$ concentration is not analysed breath by breath. FRC and ventilation distribution can be measured with the multiple-breath $\mathrm{N}_{2}$ washout. However, MBNW using $100 \% \mathrm{O}_{2}$ as a rebreathing gas should be avoided, as rebreathing pure $\mathrm{O}_{2}$ changes the breathing pattern during the measurement [15]. Although MBNW is simple to perform in spontaneously breathing infants, changes in gas viscosity and travelling time of the sample gas to the $\mathrm{N}_{2}$ analyser will significantly affect the accuracy of the measurement [23]. $\mathrm{SF}_{6}$ washout is a potentially suitable technique for infants, including those requiring near $100 \% \mathrm{O}_{2}$ to maintain normal haemoglobin saturation. $\mathrm{SF}_{6}$ concentration has previously been measured using an infrared analyser, however these analysers have a low signal-to-noise ratio and variable response times [6, 7]. $\mathrm{SF}_{6}, \mathrm{He}$ and $\mathrm{N}_{2}$ may be measured by a mass spectrometer with high precision, but the equipment is bulky and requires experienced staff to operate it. The gas dilution technique using an ultrasonic flow meter is unique, as $V^{\prime}$ and $\mathrm{MM}$ can be measured simultaneously. Flow is determined by measuring the transit time of a pulsed ultrasound travelling through the streaming medium. The MM $\left(\mathrm{g} \cdot \mathrm{mol}^{-1}\right)$ can subsequently be computed from the transit time with high accuracy and its value is directly proportional to the density of the medium. The ultrasonic flow sensor has several advantages over previous washin/washout techniques. Flow and gas concentrations are measured simultaneously and no cumbersome calibration and 
adjustment of the delay time between flow and tracer gas signal is necessary. Any inert gas with a different $\mathrm{MM}$ from air $\left(28.85 \mathrm{~g} \cdot \mathrm{mol}^{-1}\right)$ such as $\mathrm{SF}_{6}$ $\left(146 \mathrm{~g} \cdot \mathrm{mol}^{-1}\right)$ or $\mathrm{He}\left(4 \mathrm{~g} \cdot \mathrm{mol}^{-1}\right)$ can be used as a tracer gas. Further, the same equipment can be used during both spontaneous breathing and mechanical ventilation [8]. The $\mathrm{SF}_{6}$ technique is therefore highly suitable for measuring FRC and ventilation distribution.

The FRC values of the healthy infants in the present study were significantly lower than the calculated predicted values measured by the $\mathrm{He}$ dilution technique [19] (Table 2). Some but not all difference may be explained by the different Bunsen coefficients (solubility) of $\mathrm{He}$ and $\mathrm{SF}_{6}\left(\alpha_{\mathrm{SF} 6}=0.0086\right.$ and $\alpha_{\mathrm{He}}=0.0068$ respectively, $\left.\alpha_{\mathrm{SF} 6} / \alpha_{\mathrm{He}}=0.79\right)$, which results in higher $\mathrm{He}$ reabsorbtion of the lungs and may lead an overestimation of FRC by $1.2 \%$ compared to $\mathrm{SF}_{6} . \mathrm{SF}_{6}$ has a 36-fold higher $\mathrm{MM}$ than $\mathrm{He}\left(146 \mathrm{~g} \cdot \mathrm{mol}^{-1}\right.$ and $4 \mathrm{~g} \cdot \mathrm{mol}^{-1}$ respectively). Infants exhale to the closing volume of the lung during tidal breathing. It can be speculated, that more SF6 is trapped into these dependent parts of the lungs than $\mathrm{He}$, resulting in underestimation of FRC. Furthermore previous studies with a $\mathrm{He}$ or $\mathrm{N}_{2}$ dilution technique used a much higher instrumental dead space, which may result in artifactually high FRC values. However, these differences are not of clinical relevance. In contrast to $\mathrm{MBNW}$ with $100 \% \mathrm{O}_{2}$ [15] the $\mathrm{SF}_{6}$ washin/washout did not change the breathing pattern of the investigated subjects with the measured $V \mathrm{~T}$ remaining constant during the $\mathrm{SF}_{6}$ washin/ washout. The different variability of the measured volumes in the lung model $(1.3 \%)$ and the healthy infants $(5.5 \%)$ may be explained by the irregular breathing patterns of infants. The variability of the measured FRC in the present study's healthy infants is similar to values reported in the literature and well within acceptable limits for infant lung function testing [7, 15].

There are only limited values of normal distribution indices reported in the literature (Table 3). The present authors previously calculated AMDN, MDN and LCI of the MBNW in spontaneous breathing infants with lung disease [15]. A low value of any index indicates even ventilation. AMDN discriminates between the healthy infants of the present study and the previously described infants with lung disease. PCD was higher than the values reported by VILSTRUP et al. (healthy preterm infants) [7] but was lower than values measured in ventilated children with lung disease [8]. Bronchial reactivity studies in infants with lung disease are needed to accurately quantify the sensitivity of the reported distribution indices.

\section{Conclusion}

Most of the current methods to measure functional residual capacity use either the $\mathrm{N}_{2}$ washout or the He dilution techniques in infants with lung disease. These techniques are only available for research purposes and are not commercially available. The all-in-one ultrasonic flow meter is highly suitable to measure functional residual capacity and ventilation distribution since flow, volume and gas concentration can be measured simultaneously using a single signal of a pulsed ultrasound and no cumbersome calibration and signal adjustment prior to the measurement is necessary. The present authors have demonstrated that such a technique has acceptable accuracy and has a good reproducibility in vitro as well as in vivo. A complete set of measurements can be easily obtained during regular and unsedated sleep in healthy infants. The breathing pattern of the investigated infants was unaffected by the measurement technique. Therefore a new noninvasive technique has been developed that can easily assess ventilation inhomogeneities and lung volumes, a potential sensitive marker of small airway disease in unsedated infants. This makes the technique useful for large epidemiological studies in healthy infants or for uncomplicated use in a clinical setting.

\section{References}

1. Hayden MJ, Wildhaber JH, LeSouef PN. Bronchodilator responsiveness testing using raised volume forced expiration in recurrently wheezing infants. Pediatr Pulmonol 1998; 26: 35-41.

2. Frey U, Makkonnen K, Wellman T, Beardsmore C, Silverman $M$. Alterations in airwall properties in infants with history of wheezing disorders. Am J Respir Crit Care Med 2000; 161: 1825-1829.

3. Saidel GM, Saniie J, Chester EH. Modelling and moments of multibreath lung washout. Ann Biomed Eng 1978; 6: 126-137.

4. Saidel GM, Saniie J, Chester EH. Lung washout during spontaneous breathing: parameter estimation with a time-varying model. Comput Biomed Res 1980; 13: 446-457.

5. Schaanning CG, Gulsvik A. Accuracy and precision of helium dilution technique and body plethysmography in measuring lung volumes. J Clin Lab Invest 1973; 32: 271-277.

6. Schulze A, Schaller P, Töpfer A, Kirplani $H$. Measurement of functional residual capacity by sulfur hexafluoride in small-volume lungs during spontaneous breathing and mechanical ventilation. Pediatr Res 1994; 35: 494- 499.

7. Vilstrup CT, Björklund J, Larsson A, Lachmann B, Werner O. Functional residual capacity and ventilation homogeneity in mechanically ventilated small neonates. J Appl Physiol 1992; 73: 276-283.

8. Schibler A, Henning R. Measurement of functional residual capacity in rabbits and children using an ultrasonic flow meter. Pediatr Res 2001; 49: 581-588.

9. Fowler WS, Cornish ER, Kety SS. Lung function studies. VIII. Analysis of alveolar ventilation by pulmonary $\mathrm{N}_{2}$ clearance curves. J Clin Invest 1952; 31: 40-50.

10. Habib RH, Lutchen KR. Moment analysis of multiple nitrogen washout based on an alveolar gas dilution number. Am Rev Respir Dis 1991; 144: 513-519.

11. Edelman NH, Mittman C, Norris AH, Shock NW. Effects of respiratory pattern on age differences in ventilation uniformity. J Appl Physiol 1968; 24: 49-53.

12. Frey U, Reinmann B, Stocks J. The infant lung model: A mechanical analog to test infant lung function equipment. Eur Respir $J$ 2001; 17: 755-764.

13. Schuessler TF, Bates JHT. A computer-controlled 
research ventilator for small animals: Design and evaluation. IEEE Trans Biomed Eng 1995; 42: 860-866.

14. Altman DG, Bland JM. Measurement in medicine: the analysis of method comparison studies. Statistician 1983; 32: 307-317.

15. Schibler A, Schneider M, Frey U, Kraemer R. Moment ratio analysis of multiple breath nitrogen washout in infants with lung disease. Eur Respir $J$ 2000; 15: 1094-1101.

16. Sivan Y, Deakers TW, Newth CJL. An automated bedside method for measuring functional residual capacity by $\mathrm{N}_{2}$ washout in mechanically ventilated children. Pediatr Res 1990; 28: 446-450.

17. Frey U, Stocks J, Sly P, Bates JHT. Standards for infant respiratory function testing: Specifications for signal processing and data handling used in infant pulmonary function testing. Eur Respir J 2000; 16: 1016-1022.

18. Frey U, Stocks J, Coates A, Sly P, Bates JHT. Standards for infant respiratory function testing: Specifications for equipment used for infant pulmonary function testing. Eur Respir J 2000; 16: 731-740.
19. Tepper RS, Merth IT, Newth CJL, Gerhardt T. Measurement of functional residual capacity in infants by helium dilution and nitrogen washout techniques. In: Stocks J, Sly PD, Tepper RS, Morgan WJ, eds. Infant respiratory function testing. New York, John Wiley Sons, Inc., 1996; pp. 165-189.

20. Schibler AS, Henning R. Positive end-expiratory pressure and ventilation inhomogeneity in mechanically ventilated children. Ped Crit Care Med 2002; 3: 124-128.

21. Kraemer R, Meister B. Fast real-time moment-ratio analysis of multibreath nitrogen washout in children. J Appl Physiol 1985; 59: 1137-1144.

22. Shao H, Sanberg K, Sjöqvist BA, Hjalmarson O. Moment analysis of multibreath nitrogen washout in healthy preterm infants. Pediatr Pulmonol 1998; 25: 52-58.

23. Brunner JX, Wolff G, Cumming G, Langenstein $H$. Accurate measurement of $\mathrm{N}_{2}$ volumes during $\mathrm{N}_{2}$ washout requires dynamic adjustment of delay time. J Appl Physiol 1985; 59: 1008-1012. 